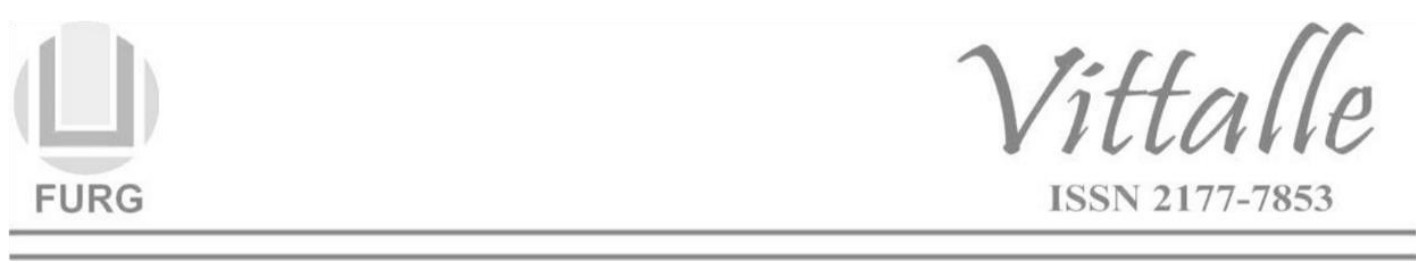

\title{
Incidência da Doença Meningocócica no Rio Grande do Sul entre 2014 a 2018
}

\author{
Adriana Heuert, Ana Lise Barbosa Soares, Marcelle Moura Silveira* \\ Curso de Licenciatura em Ciências Biológicas, Instituto Federal Farroupilha Campus Júlio de Castilhos, \\ Júlio de Castilhos, RS, Brasil
}

\section{Histórico do Artigo Recebido em: 20/09/2019 \\ Aceito em: \\ $23 / 03 / 2020$ \\ Palavras-chave: Sorogrupo Y; Rio Grande do Sul; Neisseria meningitidis}

Keywords: Serogroup Y; Rio Grande do Sul; Neisseria meningitidis

\begin{abstract}
RESUMO
A doença meningocócica é uma doença infecciosa aguda associada à altas taxas de letalidade e morbidade. Em 2010, o Brasil foi o primeiro país da América Latina a introduzir a vacina contra meningite $\mathrm{C}$ no Programa Nacional de Imunização e apesar dos números de casos associados à doença terem reduzido significativamente em crianças menores de 2 anos de idade, público alvo da campanha de vacinação, os sorogrupos C e B ainda são os mais prevalentes no Brasil. O objetivo deste trabalho foi avaliar a incidência dos sorogrupos associados à doença meningocócica no Rio Grande do Sul. Os dados referentes à doença durante o período de 2014 a 2018 foram coletados do Sistema Nacional de Agravos de Notificação. Nossos resultados demonstraram pela primeira vez que no Rio Grande do Sul o sorogrupo Y é o responsável pela maior incidência da doença meningocócica.
\end{abstract}

\section{Incidence of Meningococcal Disease in Rio Grande do Sul between 2014 to 2018}

\begin{abstract}
Meningococcal disease is an acute infectious disease associated with high mortality and morbidity rates. In 2010, Brazil was the first country in Latin America to introduce the meningitis $\mathrm{C}$ vaccine in the National Immunization Program. Although the number of cases associated with the disease significantly decreased in children under 2 years old, target of the campaign vaccination, serogroups $\mathrm{C}$ and $\mathrm{B}$ are still the most prevalent in Brazil. The objective of this study was to evaluate the incidence of serogroups associated with meningococcal disease in Rio Grande do Sul. Data regarding the disease during the period from 2014 to 2018 were collected from the National System of Notification Disorders. Our results demonstrated for the first time that in Rio Grande do Sul, serogroup $\mathrm{Y}$ is responsible for the highest incidence of meningococcal disease.
\end{abstract}

\section{Introdução}

A doença meningocócica (DM) é uma infecção grave causada pela Neisseria meningitidis, bactéria Gram-negativa em forma de coco, imóvel e aeróbia, que resulta em um amplo espectro clínico, incluindo meningite, meningococcemia ou ambas, sendo a meningite a forma clínica mais frequentemente observada (1-3). A DM é um importante problema de saúde mundial que tem aparecimento súbito, progride rapidamente, tem uma alta taxa de letalidade e uma proporção considerável de sobreviventes e têm sequelas incapacitantes permanentes, tais como surdez, deficiências neurológicas e amputação $(2,4,5)$.

Os meningococos são divididos em 13 grupos sorológicos, com base na antigenicidade de seus polissacarídeos capsulares. Os sorotipos A, B, C, Y, W e X causam a maioria dos casos de meningite e meningococcemia $(6,7)$. $\mathrm{O}$ meningococo é transmitido de pessoa a

\footnotetext{
*Autor correspondente: marcellemsilveira@gmail.com (Silveira M.M.)
} 
pessoa através de contato com secreções respiratórias ou por inalação de gotículas em aerossóis $(1,7)$. A DM acomete indivíduos de todas as faixas etárias, mas a incidência é mais elevada entre as crianças menores de cinco anos de idade. Entretanto, durante as epidemias e em situações de surto, a incidência da doença tende a aumentar principalmente entre crianças mais velhas, adolescentes e adultos jovens (3).

No Brasil, os sorogrupos B e C são os responsáveis pela alta incidência da DM $(8,9)$. Infecções com o sorogrupo C têm sido notificadas desde 2005 com taxas de letalidade em torno de $20 \%$, sendo as taxas de incidência mais elevadas entre crianças menores de quatro anos de idade $(8,10)$. Em 2010, a introdução da vacina conjugada contra o meningococo $\mathrm{C}$ (MenC) no Programa Nacional de Imunizações (PNI) para crianças menores de 2 anos, forneceu uma redução imediata nas taxas de incidência da doença entre os grupos etários-alvo para a vacina no Brasil $(11,12)$. A vacinação é uma forma barata e eficaz de reduzir a inequidade na saúde. O monitoramento da DM é crucial para melhorar a compreensão da epidemiologia da doença, bem como para identificação de políticas ótimas de vacinação e controle da meningite. Assim, o objetivo do presente trabalho foi avaliar a DM no Rio Grande do Sul, entre os anos de 2014 e 2018, bem como a contribuição de cada sorogrupo na carga total de doença.

\section{Materiais e métodos}

Foi conduzido um estudo descritivo com análise da incidência de cada sorotipo de DM no estado do Rio Grande do Sul durante o período de 2014 a 2018. A pesquisa foi desenvolvida utilizando bases de dados secundários.

Dados epidemiológicos, casos notificados e/ou confirmados da DM são registrados no Sistema Nacional de Agravos de Notificação (SINAN) (13). Os dados secundários referentes à ocorrência dos casos de DM no Rio Grande do Sul foram coletados na base de dados do Sistema Nacional de Vigilância Epidemiológica do Ministério da Saúde e Departamento de Informática do SUS (DATASUS; http://www.datasus.gov.br).

Os dados populacionais foram obtidos no Programa Nacional de Amostra a domicílio (PNAD). A incidência para cada sorotipo foi calculada como o número de casos novos da doença notificados ao SINAN dividido pela população residente no Rio Grande do Sul em cada ano, multiplicado por 100.000 habitantes. Em relação aos números e casos novos, foram considerados apenas os casos em que a doença foi confirmada no mesmo ano em que os primeiros sintomas foram relatados.

\section{Resultados e discussão}

Este estudo avaliou a incidência da DM no Rio Grande do Sul, bem como a contribuição de cada sorogrupo na carga da doença entre os anos de 2014 e 2018. Durante esse período, foram registrados 5.926 casos da DM no estado do Rio Grande do Sul. A maior incidência da doença ocorreu em 2017 seguido por 2015 onde 1.321 e 1.269 casos foram notificados, respectivamente. No ano de 2016 foram registrados 1.070 casos com uma menor incidência de 9,4 casos por 100.000 habitantes.

Uma epidemia da DM no Rio Grande do Sul ocorreu em 1974 onde foi reportado uma incidência de 40,8 casos por 100.000 habitantes (14). No período de 1983 a 1992 foi descrita uma incidência de 1,3 casos por 100.000 habitantes e em 1993 houve um aumento para 2,5 casos por 100.000 habitantes (14). Além disso, desde 2004 tem sido reportado um aumento no número de casos de DM associada ao sorogrupo $\mathrm{W}$ na região Sul do Brasil, onde o número de casos aumentou de 3,2\% entre o período de 1995 a 2003 para $17,8 \%$ dos casos entre 2003 a 2005 (15). 
De 1995 a 2003, 69.8\% dos casos da DM foram atribuídas ao sorogrupo B no Rio Grande do Sul (14). Em nosso trabalho nós avaliamos a incidência da doença entre os anos de 2014 e 2018 e o sorogrupo $\mathrm{C}$ foi predominante (Figura 1) com incidências de 5,8; 7,0; 6,9; 8,3 e 6,3 casos por 100.000 habitantes, respectivamente. Em 2010, o Brasil foi o primeiro país da América Latina a incluir a vacina contra MenC no PNI (3). No primeiro ano de vida são recomendadas duas doses da vacina meningocócica $\mathrm{C}$ conjugada, aos $3 \mathrm{e}$ 5 meses. A dose de reforço deve ser administrada entre 12 e 15 meses de idade. A introdução da vacina contra o sorogrupo $\mathrm{C}$ reduziu imediatamente a incidência da doença entre as crianças com idade alvo para vacinação no Brasil (16).

De acordo com a Organização Pan-Americana da Saúde em 2012, a maioria dos casos de DM ocorridos no Brasil foram atribuídas ao sorogrupo $\mathrm{C}$ seguido pelo sorogrupo $\mathrm{B}$ (17). Entretanto, no Rio Grande do Sul, o sorogrupo W foi o segundo sorogrupo mais associado a DM nos anos de 2014 e 2016 (Tabela 1) com incidência de 2.2 e 1.7 casos por 100.000 habitantes, respectivamente (Figura 1).

Tabela 1 - Percentual de cada sorogrupo associado a DM no Rio Grande do Sul

\begin{tabular}{cccccc}
\hline & \multicolumn{5}{c}{ Sorogrupos (\%) } \\
\cline { 2 - 6 } Anos & $\mathrm{B}$ & $\mathrm{C}$ & $\mathrm{W}$ & $\mathrm{X}$ & $\mathrm{Y}$ \\
\hline 2014 & 20 & 58 & 22 & $\mathrm{NR}$ & $\mathrm{NR}$ \\
2015 & 23 & 62 & 15 & $\mathrm{NR}$ & $\mathrm{NR}$ \\
2016 & 7 & 72 & 18 & 3 & $\mathrm{NR}$ \\
2017 & 10 & 74 & 10 & 2 & 4 \\
2018 & 22 & 63 & 9 & $\mathrm{NR}$ & 6 \\
\hline NR- não relatado. & & & & &
\end{tabular}

Nossos dados demonstram pela primeira vez o aumento da incidência da DM associado ao sorogrupo Y no estado do Rio Grande do Sul. Um aumento na incidência da doença associada a esse sorogrupo tem sido reportado na América Latina durante o período de 2000 a 2006, variando de 3,6\% para 50\% dos casos na Colômbia. Além disso, um aumento nos casos do sorogrupo Y também foi encontrado na Venezuela (18).

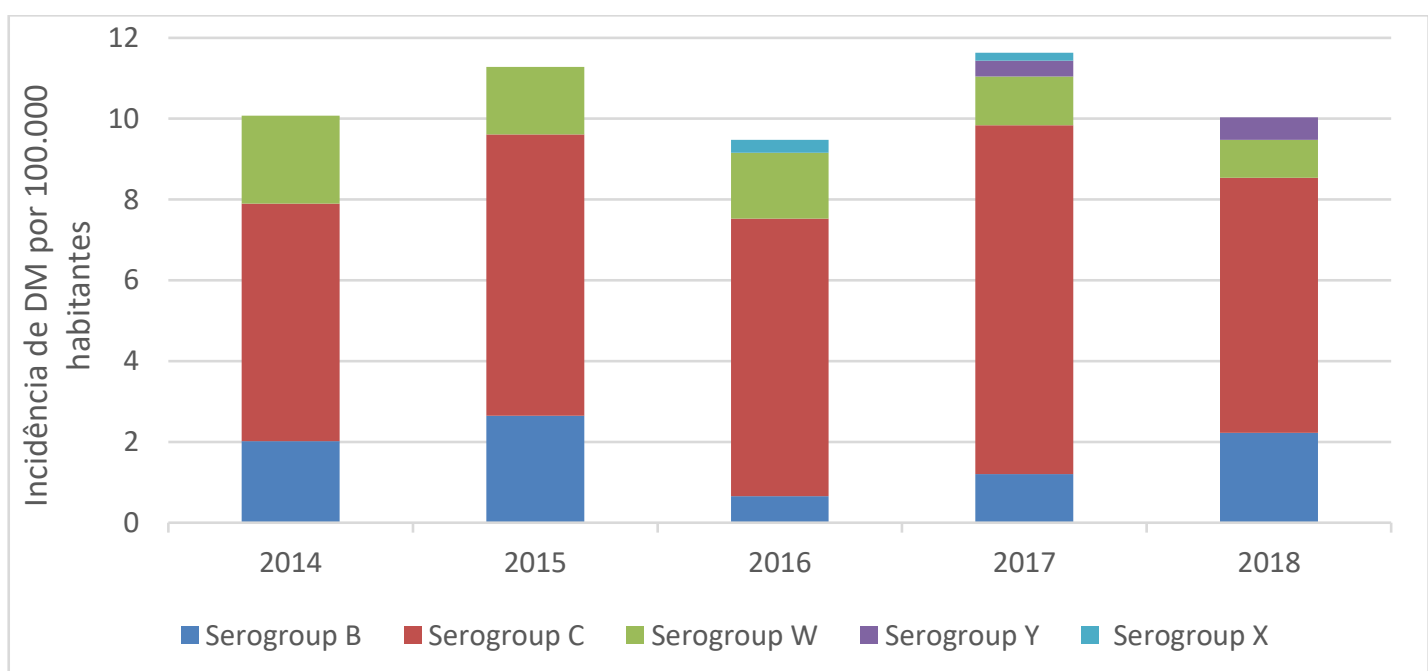

Figura 1 - Incidência dos sorotipos B, C, W, X e Y associados à DM no Rio Grande do Sul no período de 2014 a 2018. 
A vacinação tem contribuído de forma eficaz no controle de muitas doenças infecciosas e é considerada uma das maiores descobertas na área médica nas últimas décadas (19). Além da vacina contra menC, que atualmente é fornecida gratuitamente no PNI, a vacina contra menB (Bexsero) foi licenciada no Brasil em 2015, entretanto, não foi incluída no PNI. Recentemente, a vacina quadrivalente (ACWY) conjugada que fornece proteção contra os sorogrupos A, C, W e Y também foi licenciada no Brasil e devido ao aumento na incidência do sorogrupo $\mathrm{W}$ em algumas regiões do país, essa vacina deverá ser incluída no PNI. A vacina quadrivalente conjugada (ACWY) é utilizada atualmente nos Estados Unidos e no Canada sendo administrada a adolescentes com 11 a 12 anos de idade e uma dose de reforço é realizada após 5 anos. Além disso, os adolescentes com idade entre 13 e 18 anos que não receberam previamente a vacina também devem ser imunizados.

As vacinas meningócocicas conjugadas (A, C ou ACWY), associada à vacina recombinante contra o sorogrupo B estão disponíveis e oferecem uma possibilidade de controle mundial da DM. É importante considerarmos, entretanto, que são necessários estudos sorológicos para avaliarem o tempo de proteção conferido por essas vacinas e quando devem ser administradas as doses de reforço. Esses estudos sororógicos são importantes principalmente no que se refere à vacina Bexsero contra o sorogrupo $\mathrm{B}$, a qual as análises de custo-efetividade que foram realizadas em diversos países assumiram diferentes tempos de proteção (20-24).

Além disso, a escolha das vacinas que são incluídas no PNI deve considerar a epidemiologia da doença em cada região. Com esse estudo demonstramos um aumento na incidência da DM no estado do Rio Grande do Sul associada aos sorogrupos Y e W. A inclusão da vacina quadrivalente (ACWY) conjugada pode permitir a redução das mortes e sequelas severas ocasionadas pela DM. Em nosso trabalho demonstramos também a importância de considerar fatores regionais associados às doenças infecciosas $(18,25)$. As escolhas adequadas das vacinas a serem fornecidas no PNI é uma forma barata e eficaz de reduzir a inequidade na saúde.

\section{Referências}

1. Safadi MA, Berezin EN, Oselka GW. A critical appraisal of the recommendations for the use of meningococcal conjugate vaccines. J Pediatr (Rio J) 2012;88(3):195-202.

2. Bastos RC, de Souza IM, da Silva MN, Silva FP, Figueira ES, Leal ML, et al. Brazilian meningococcal C conjugate vaccine: Scaling up studies. Vaccine 2015; 33 (35): 4281-4287.

3. Cardoso CW, Ribeiro GS, Reis MG, Flannery B, Reis JN. Effectiveness of meningococcal C conjugate vaccine in salvador, Brazil: a case-control study. PLoS One. 2015; 10(4) :e0123734.

4. Christensen H, Trotter CL, Hickman M, Edmunds WJ. Re-evaluating cost effectiveness of universal meningitis vaccination (Bexsero) in England: modelling study. Bmj. 2014; 349: g5725.

5. Safadi MA, Bettinger JA, Maturana GM, Enwere G, Borrow R. Evolving meningococcal immunization strategies. Expert Rev Vaccines 2015; 14(4): 505-17.

6. Brehony C, Trotter CL, Ramsay ME, Chandra M, Jolley KA, van der Ende A, et al. Implications of differential age distribution of disease-associated meningococcal lineages for vaccine development. Clin Vaccine Immunol 2014; 21(6): 847-53.

7. Trotter CL, Maiden MC. Meningococcal vaccines and herd immunity: lessons learned from serogroup C conjugate vaccination programs. Expert Rev Vaccines 2009; 8(7): 851-61.

8. Tauil Mde C, Carvalho CS, Vieira AC, Waldman EA. Meningococcal disease before and after the introduction of meningococcal serogroup C conjugate vaccine. Federal District, Brazil. The Brazilian journal of infectious diseases : an official publication of the Brazilian Society of Infectious Diseases 2014; 18(4): 379-86.

9. de Filippis I, de Lemos AP, Hostetler JB, Wollenberg K, Sacchi CT, Dunning Hotopp JC, et al. Molecular epidemiology of Neisseria meningitidis serogroup B in Brazil. PloS one. 2012; 7(3): e33016. 
10. Azevedo LC, Toscano CM, Bierrenbach AL. Bacterial Meningitis in Brazil: Baseline Epidemiologic Assessment of the Decade Prior to the Introduction of Pneumococcal and Meningococcal Vaccines. PLoS One. 2013; 8(6): e64524.

11. Lucidarme J, Hill DM, Bratcher HB, Gray SJ, du Plessis M, Tsang RS, et al. Genomic resolution of an aggressive, widespread, diverse and expanding meningococcal serogroup B, C and W lineage. J Infect 2015; 71(5): 544-52.

12. de Moraes JC, Kemp B, de Lemos AP, Gorla MC, Marques EG, Ferreira MD, et al. Prevalence, Risk Factors and Molecular Characteristics of Meningococcal Carriage Among Brazilian Adolescents. Pediatr Infect Dis J 2015; 34(11): 1197-202.

13. Cardoso CW, Pinto LL, Reis MG, Flannery B, Reis JN. Impact of vaccination during an epidemic of serogroup C meningococcal disease in Salvador, Brazil. Vaccine 2012; 30(37): 5541-6.

14. Baethgen LF, Weidlich L, Moraes C, Klein C, Nunes LS, Cafrune PI, et al. Epidemiology of meningococcal disease in southern Brazil from 1995 to 2003, and molecular characterization of Neisseria meningitidis using multilocus sequence typing. Tropical medicine \& international health : TM \& IH 2008; 13(1): 31-40.

15. Weidlich L, Baethgen LF, Mayer LW, Moraes C, Klein CC, Nunes LS, et al. High prevalence of Neisseria meningitidis hypervirulent lineages and emergence of W135:P1.5,2:ST-11 clone in Southern Brazil. The Journal of infection 2008; 57(4): 324-31.

16. Safadi MA, de los Monteros LE, Lopez EL, Saez-Llorens X, Lemos AP, Moreno-Espinosa S, et al. The current situation of meningococcal disease in Latin America and recommendations for a new case definition from the Global Meningococcal Initiative. Expert review of vaccines 2013; 12(8): 903-15.

17. Batista RS, Gomes AP, Dutra Gazineo JL, Balbino Miguel PS, Santana LA, Oliveira L, et al. Meningococcal disease, a clinical and epidemiological review. Asian Pacific journal of tropical medicine 2017; 10(11): 1019-29.

18. Safadi MA, Cintra OA. Epidemiology of meningococcal disease in Latin America: current situation and opportunities for prevention. Neurological research 2010; 32(3): 263-71.

19. Silveira MM, Oliveira TL, Schuch RA, McBride AJA, Dellagostin OA, Hartwig DD. DNA vaccines against leptospirosis: A literature review. Vaccine 2017; 35(42): 5559-67.

20. Christensen H, Irving T, Koch J, Trotter CL, Ultsch B, Weidemann F, et al. Epidemiological impact and cost-effectiveness of universal vaccination with $\operatorname{Bexsero}((\mathrm{R}))$ to reduce meningococcal group $\mathrm{B}$ disease in Germany. Vaccine 2016; 34(29): 3412-9.

21. Tu HA, Deeks SL, Morris SK, Strifler L, Crowcroft N, Jamieson FB, et al. Economic evaluation of meningococcal serogroup B childhood vaccination in Ontario, Canada. Vaccine 2014; 32(42): 5436-46.

22. Tirani M, Meregaglia M, Melegaro A. Health and economic outcomes of introducing the new MenB vaccine (Bexsero) into the Italian routine infant immunisation programme. PloS one. 2015; 10(4): e0123383.

23. Pouwels KB, Hak E, van der Ende A, Christensen H, van den Dobbelsteen GP, Postma MJ. Costeffectiveness of vaccination against meningococcal B among Dutch infants: Crucial impact of changes in incidence. Hum Vaccin Immunother 2013; 9(5): 1129-38.

24. Lecocq H, Parent du Chatelet I, Taha MK, Levy-Bruhl D, Dervaux B. Epidemiological impact and cost-effectiveness of introducing vaccination against serogroup B meningococcal disease in France. Vaccine 2016; 34(19): 2240-50.

25. de Soarez PC, Sartori AM, de Andrade Lagoa Nobrega L, Itria A, Novaes HM. Cost-effectiveness analysis of a universal infant immunization program with meningococcal C conjugate vaccine in Brazil. Value in health : the journal of the International Society for Pharmacoeconomics and Outcomes Research 2011; 14(8):1019-27. 